\title{
Spin dynamics of carriers in GaAs quantum wells in an external electric field
}

\author{
I. Ya. Gerlovin,* Yu. K. Dolgikh, S. A. Eliseev, and V. V. Ovsyankin \\ Vavilov State Optical Institute, St.-Petersburg 190034, Russia \\ Yu. P. Efimov, I. V. Ignatiev, V. V. Petrov, and S. Yu. Verbin \\ Institute of Physics, St.-Petersburg State University, St.-Petersburg 198904, Russia \\ Y. Masumoto \\ Institute of Physics, University of Tsukuba, Tsukuba 305-8571, Japan
}

(Received 22 September 2003; published 28 January 2004)

\begin{abstract}
The effect of external electric bias on the kinetics of circularly polarized photoluminescence (PL) of the GaAs quantum wells (QWs) is studied experimentally. It is found that a negative bias applied to the top surface of the samples causes the appearance of a slow component in the decay of the PL circular polarization. The amplitude of this component grows with the bias $U_{\text {bias }}$ and reaches nearly $100 \%$ at $U_{\text {bias }}=-2 \mathrm{~V}$. In a transverse magnetic field, the polarization decay shows oscillations related to the spin precession. The changes in the shape of the oscillations with the applied bias indicate a transition from the exciton spin precession to that of the electron spin. Based on the analysis of the experimental data, we came to the conclusion that the external electric field reduces the exchange coupling between the electron and hole spins. As a result, the hole spin exhibits fast relaxation whereas the electron spin holds its light-induced orientation for a relatively long time. The studies of the electron spin dynamics in oblique magnetic fields have allowed us to estimate the effective energy of the electron-hole exchange interaction in the electric field created by the bias $U_{\text {bias }}=-2 \mathrm{~V}$. The exchange splitting thus obtained is much smaller than typical exchange splittings of the excitonic states in the GaAs QWs.
\end{abstract}

DOI: $10.1103 /$ PhysRevB.69.035329

PACS number(s): 73.21.-b, 75.30.Et, 71.70.-d, 78.55.-m

\section{INTRODUCTION}

In the last decade, considerable interest has arisen in studies of spin states in semiconductor heterostructures. A great deal of attention is now attracted to feasibility of spin transistors, ${ }^{1}$ spin logic switches, and elements of spin memory for quantum computers. ${ }^{2,3}$ Intense research is devoted to studying the relaxation of spins of individual carriers (electrons and holes) or of the electron-hole pairs (excitons) coupled by the Coulomb interaction. An important role in the exciton spin dynamics is played by exchange interaction that couples the electron and hole spins. This interaction is distinctly revealed in the spin relaxation rates. In particular, while the relaxation time of the free-hole spin in the GaAs- based heterostructures lies, according to Ref. 4, in the range of units of picoseconds and the electron-spin relaxation time may reach $1000 \mathrm{ps}$ and more, ${ }^{5-7}$ the exciton spin is characterized by intermediate values of the relaxation times lying in the range of 100-200 ps. ${ }^{8,9}$ This is, in fact, a result of competition between the exchange coupling, correlating the motion of the electron and hole spins, and the perturbation inducing the spin relaxation of individual carriers (primarily, of hole). Indeed, as shown in Ref. 10, in the narrow GaAs quantum wells, below $5 \mathrm{~nm}$ thick, where the exchange interaction is large, and the relaxation of the hole spin is strongly suppressed, ${ }^{11}$ the spin dynamics is well described by the motion of the exciton spin as a whole. In the layers thicker than $20 \mathrm{~nm}$, the spin-phonon interaction for the hole exceeds the exchange one. As a result, the hole spin rapidly relaxes, while the electron spin holds its orientation much longer than the total spin of the exciton. ${ }^{12,13}$ In the quantum wells about $10 \mathrm{~nm}$ thick, the situation appears to be intermediate, with the energies of the exchange and spinphonon coupling being of the same order of magnitude. This fact offers a fundamentally new opportunity to control the exciton spin relaxation rate by varying the ratio between these parameters.

It seems highly probable that the competition between the exchange and spin-phonon interaction is responsible for the paradoxical effect described in Ref. 14. The effect consists in slowing down, rather than accelerating, of the spin quantum beats decay with increasing temperature. Most likely, this effect is due to decoupling of the electron and hole spins at elevated temperatures. As a result, the electron spin can freely precess for a sufficiently long time. The electron-spin free precession has been also detected in Ref. 10 upon excitation of the photoluminescence (PL) with a large Stokes shift. According to Ref. 10, the structure is locally heated in the course of the excitation relaxation. The results of these experiments show that the opportunity to control the electron spin dynamics is quite realistic. At the same time, such an inertial and hard-to-control parameter as local temperature can hardly be used for this purpose.

We think that a more straightforward way to affect the exciton spin relaxation is to vary the electron-hole exchange interaction by applying an electric field normal to the heterostructure layers. The field polarizes the exciton along the growth axis, thus decreasing the overlap of the electron and hole wave functions. ${ }^{15-17}$ According to theoretical estimates made in Ref. 16, the exchange coupling in the GaAs quan- 
tum wells $10-15 \mathrm{~nm}$ thick shows a noticeable decrease in the fields about $100 \mathrm{kV} / \mathrm{cm}$. The electric field of this strength can be produced by the bias of about $10 \mathrm{~V}$ applied to the structure, which can be easily realized under usual experimental conditions.

In this paper, we present the results of studying the effect of electric field on kinetics of the polarized excitonic PL of heterostructures with the GaAs quantum wells $10 \mathrm{~nm}$ or less in thickness. We have found that the application of the external electric bias not only drastically changes the spin relaxation rates, but also substantially affects the spin precession in the external magnetic field. The analysis of the experimental data has shown that the observed effects are indeed related to the breakage of the exchange coupling between the electron and hole spins.

The paper is organized as follows. A brief description of the experimental conditions is followed by two main sections. The first of them describes the effect of electric field on the kinetics of circularly polarized PL both in zero magnetic field and in a magnetic field aligned perpendicular to the growth axis of the structure (Voigt configuration). It is shown that, in all the samples under study, the kinetics of the polarized PL in the absence of the external bias is determined by dynamics of the total exciton spin. Application of an external bias noticeably changes the kinetics of the polarized PL of the samples with $10 \mathrm{~nm}$ quantum wells. The kinetics acquires the features typical for the free electron spin dynamics.

The next section is devoted to the electron spin dynamics in the magnetic fields deviated from the exact Voigt configuration. A previously unobserved mechanism of the electron spin dephasing is found. The dephasing is caused by the exchange interaction with the randomly oriented spin of the hole. The paper is concluded by a summary of the main results of the work.

\section{EXPERIMENTAL DETAILS}

We studied a set of heterostructures with single GaAs QWs (SQWs), multi-QWs (MQWs), and double QWs separated by a relatively thin $(20 \mathrm{~nm}) \mathrm{Al}_{0.3} \mathrm{Ga}_{0.7} \mathrm{As}$ barrier. The thickness of the QWs was varied from 8 to $10 \mathrm{~nm}$. In the samples with double QWs, one of the wells was doped with Si to study the effect of free electrons on the spin dynamics of the excitons. The samples were grown on the $n$-doped GaAs substrate using the MBE technique. The top surface of each sample was coated with a semitransparent ITOelectrode for application of the external bias. We measured the kinetics of the circularly polarized PL excited by short ( 2 ps) pulses of a tunable Ti-sapphire laser. The PL kinetics was detected in real time using a streak camera. The kinetics of the circularly polarized PL was studied as a function of the electric bias and of the magnitude and orientation of the external magnetic field. All the measurements were made at $5 \mathrm{~K}$.

The PL spectra of the structures under study displayed intense excitonic lines with a halfwidth of 2-3 meV. Approximately of the same value was the Stokes shift between the exciton peaks in the absorption and PL spectra. The PL was excited directly to the exciton absorption band and was detected with the small long-wavelength shift $(\approx 2 \mathrm{meV})$ to minimize the polarization losses. In all the experiments, the exciting beam was directed at an angle of $90^{\circ}$ to the magnetic field direction, and the PL was detected in the backward direction. Orientation of the magnetic field with respect to the growth axis of the structure was changed by rotating the sample. In this case, the angle of incidence of the light on the sample was changed but, due to the large refractive index of the medium, the light was propagating inside the sample practically along the growth axis even at relatively large rotation angles (up to $50^{\circ}$ ).

\section{DYNAMICS OF THE DEGREE OF CIRCULAR POLARIZATION}

\section{A. Experimental results}

As was found in preliminary experiments, the PL spectra of the undoped QWs, in the whole range of the biases $\left(-2 \mathrm{~V} \geqslant U_{\text {bias }} \leqslant 0 \mathrm{~V}\right)$, did not show trionic lines characteristic of the QWs with excess electrons. ${ }^{18-21}$ The electric-field induced shift of the exciton peaks was found to agree well with the results of studying the Stark effect in the neutral QWs with the same thickness. ${ }^{22}$ This allowed us to conclude that the QWs under study virtually did not contain free carriers. Thus, the effect arising in the presence of the bias may result only from the action of the electric field upon the excitons. In addition, it has been established that practically all the observed regularities are critically related to the QW thickness rather than to the sample topology. In particular, the results of the measurements on the samples with MQWs coincided not only qualitatively but also quantitatively with the results obtained on the sample with single QWs of the same thickness. For this reason, we will present in the following mainly the results of studying the samples with MQWs examined in more detail.

To avoid possible spurious effects related to the circular dichroism of the optical system, we performed the measurements under both $\sigma^{+}$and $\sigma^{-}$polarized excitation. Because we did not find any noticeable dependence of the kinetics under study on the handedness of the excitation, we present here only the data obtained under $\sigma^{+}$polarized excitation.

Figure 1 demonstrates the effect of electric bias on the circularly polarized PL kinetics for two samples with the QWs 8 and $10 \mathrm{~nm}$ thick. The PL was measured in the $\sigma^{+}$and $\sigma^{-}$polarizations under $\sigma^{+}$polarized excitation in the absence of the applied magnetic field. Figure 1 shows the kinetics of the degree of circular polarization defined as the ratio of the difference of the signals of the $\sigma^{+}$and $\sigma^{-}$polarized PL to their sum. As is seen, the PL polarization kinetics of the $8 \mathrm{~nm}$ QWs is practically unaffected by the electric bias, while, in the $10 \mathrm{~nm}$ QWs, the kinetics substantially depends on the bias. In the absence of the bias, the kinetics of the PL degree of polarization of all the samples is dominated by a fast component characterized by the decay time below 100 ps. In addition, there is also a small slow component with the decay time $\sim 1$ ns. When a negative bias is applied to the top electrode of the sample with the $10 \mathrm{~nm}$ 

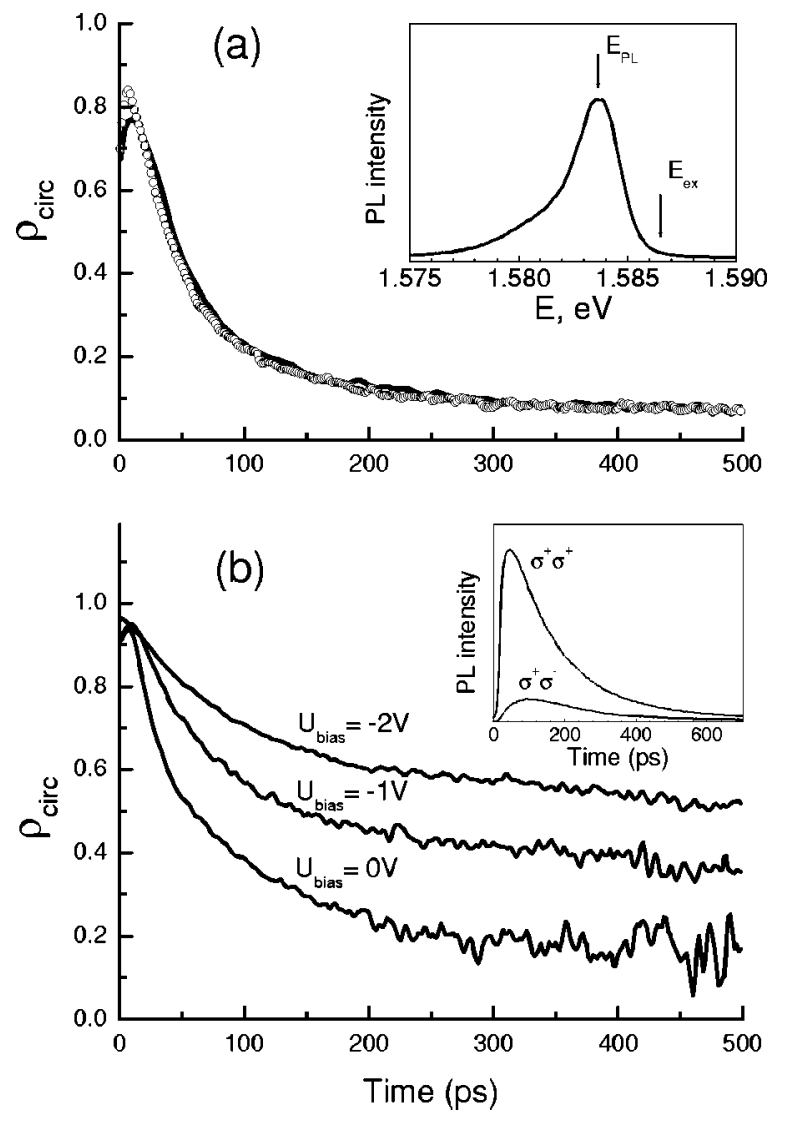

FIG. 1. The kinetics of the degree of circular polarization of the PL $\left(\rho_{\text {circ }}\right)$ with no magnetic field for different values of the bias. (a) Kinetics for $8 \mathrm{~nm}$ MQWs (sample e268) at $U_{\text {bias }}=0 \mathrm{~V}$ (solid line) and $U_{\text {bias }}=-2 \mathrm{~V}$ (open circles). Inset: the line shape of the excitonic PL. The arrows show the energies of the excitation $\left(E_{\mathrm{ex}}\right)$ and $\mathrm{PL}\left(E_{\mathrm{PL}}\right)$. (b) Kinetics for $10 \mathrm{~nm}$ MQWs (sample e274) at different biases indicated near each curve. Inset: the pulse shape of the coand cross-circular-polarized PL $\left(\sigma^{+} \sigma^{+}\right.$and $\sigma^{+} \sigma^{-}$, respectively) of the $10 \mathrm{~nm}$ QW for the bias $U_{\text {bias }}=-2 \mathrm{~V}$.

QWs, the amplitude of the slow component increases and, for the bias $U_{\text {bias }}=-2 \mathrm{~V}$, this component predominates.

The effect of electric bias on the polarized PL kinetics of the $10 \mathrm{~nm}$ QWs appears to be even more pronounced in the presence of the magnetic field oriented perpendicular to the observation direction (transverse field), as is evident from Fig. 2. For the bias $U_{\text {bias }}=-2 \mathrm{~V}$, the polarization kinetics shows distinct oscillations symmetric with respect to the $x$ axis. The oscillation amplitude is initially equal to $\rho_{0}^{\text {osc }}$ $\approx 0.75$ and slowly decays with time. The shape of the oscillations can be well fitted by the exponentially damping harmonic function

$$
\rho=\rho_{0}^{\mathrm{osc}} \exp (-t / \tau) \cos (\omega t),
$$

which allows one to find the oscillation frequency $\omega$ and the decay time $\tau$. The dependence of the oscillation frequency on the magnetic field is shown in Fig. 3(a). As is seen, the frequency is directly proportional to the magnetic field in the whole range of the field used $(0-8 \mathrm{~T})$. The decay time of the

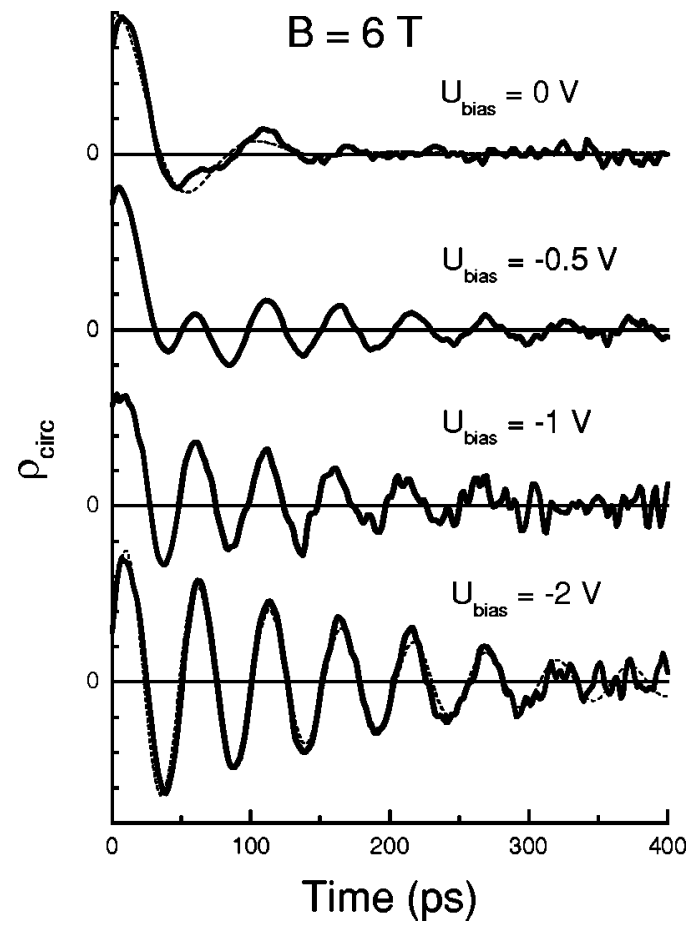

FIG. 2. The kinetics of the degree of circular polarization of the PL in the magnetic field $B=6 \mathrm{~T}$ (Voigt configurations) for different values of the bias indicated near the curves. Solid lines are the experimental data and dashed lines are the fits by Eq. (1).

oscillations in small magnetic fields is close to $1 \mathrm{~ns}$ and drops to $150 \mathrm{ps}$ in the field $B=8 \mathrm{~T}$. In the absence of the bias, the PL polarization kinetics in the magnetic field is essentially different: it is characterized by a sharp initial drop in the degree of polarization accompanied by weak rapidly damping oscillations with a complex shape [see the upper curve in Fig. 2]. By approximating the oscillations by Eq. (1), one can estimate the mean effective value of the oscillation frequency. The frequencies found in this way also grow linearly with the magnetic field [see Fig. 3(a), the lower curve], but with slope substantially smaller than in the presence of the bias. For the intermediate values of the bias voltage, the kinetics of the degree of polarization can be presented as a superposition of the rapidly and slowly damping components oscillating at different frequencies. The fraction of the slowly damping high-frequency component increases with increasing magnitude of the bias [see Fig. 2].

\section{B. Discussion}

\section{The effect of the bias}

The fine structure of the hh-exciton, responsible for the main part of the PL, comprises four sublevels with the projections of the total angular momentum on the growth axis of the structure ( $z$ axis) $J_{z}= \pm 1$ and $J_{z}= \pm 2 .{ }^{23}$ As was mentioned in Sec. II, the directions of the exciting beam and detected PL well coincide with the $z$ axis. In conformity with the selection rules, the $\sigma^{+}$polarized light will excite, in this case, only one excitonic sublevel with $J_{z}=+1$ (the hole and 

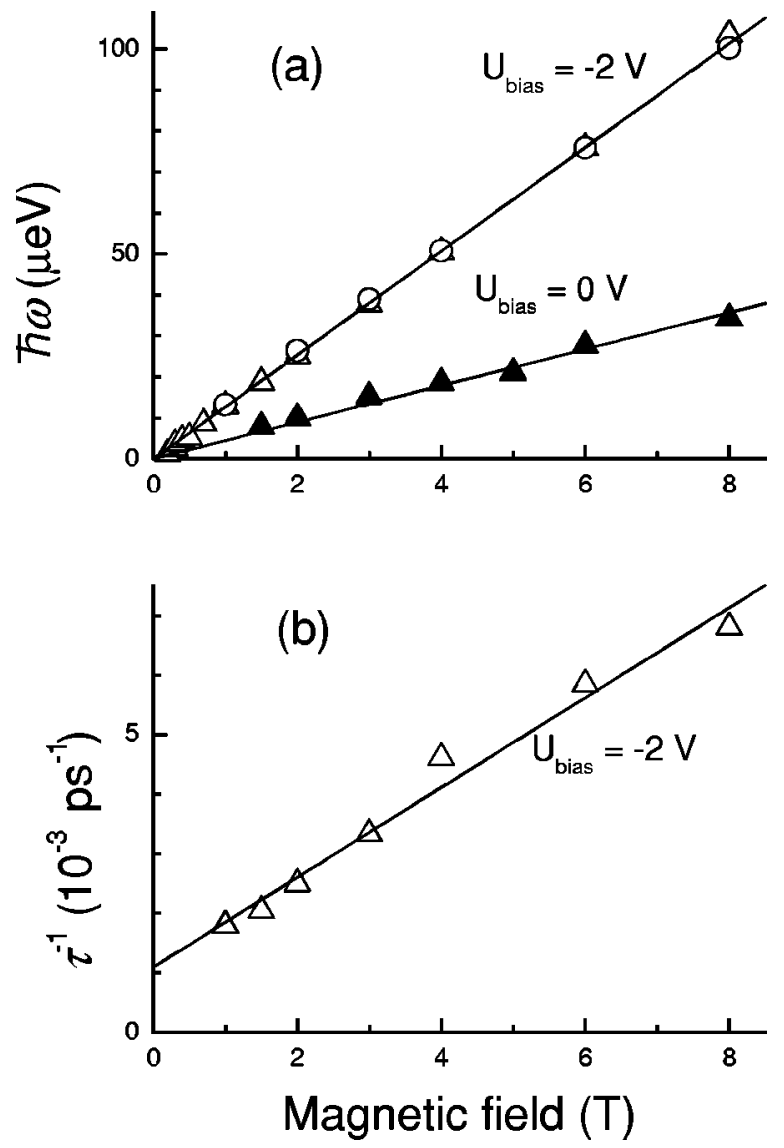

FIG. 3. Characteristics of the oscillations of the degree of circular polarization in a magnetic field (Voigt configuration). (a) Variation of the oscillation frequency $\omega$ with the magnetic field for different values of the bias indicated near the curves. Symbols are the experimental data obtained at $U_{\text {bias }}=-2 \mathrm{~V}$ for $10 \mathrm{~nm}$ MQWs (sample e274, open triangles) and SQW (sample e294, open circles) and at $U_{\text {bias }}=0 \mathrm{~V}$ for $10 \mathrm{~nm}$ MQWs (sample e274, closed triangles). Solid lines are the theoretical fittings by the formula $\hbar \omega$ $=g_{e} \mu B$. The values of the fitting parameter $g_{e}$ for each line are given in the text. (b) Dependence of the oscillation decay rate $\tau^{-1}$ on the magnetic field for sample e274 (10 nm MQW) at bias $U_{\text {bias }}=-2 \mathrm{~V}$ (open triangles). Solid line is the theoretical fitting by the formula $\tau^{-1}=\gamma_{0}+\Delta g_{e} \mu B / 2$ (see in the text) with parameters $\gamma_{0}=8.5 \times 10^{-4} \mathrm{ps}^{-1}$ and $\Delta g_{e}=0.02$.

electron spin projections are $m_{h}=+3 / 2$ and $m_{e}=-1 / 2$ ). The PL from this sublevel is also $\sigma^{+}$polarized.

Relaxation of the excitonic spin oriented by the light will result in the PL depolarization. As was pointed out earlier, the two alternative mechanisms of the spin relaxation are possible, namely, a flip of the exciton spin as a whole or independent flips of the electron and hole spins. In the first case, the decay time of the degree of polarization is directly determined by the exciton spin relaxation rate. ${ }^{16}$ If the carrier spins relax independently, the fast flip of the hole spin does not affect the PL polarization. ${ }^{16}$ The decay of the degree of polarization is controlled, in this case, by the relaxation of the long-lived electron spin.

The above treatment allows us to conclude that the biasinduced changes in the polarized PL kinetics are related to transition from dynamics of the exciton spin to that of independent electron and hole spins. In the absence of the bias, the exchange coupling exceeds the spin-phonon interaction, and the main relaxation mechanism is provided by the exciton spin flips. It is this process that is responsible for the fast component of the polarization decay of the PL in our experiments.

The electric field created by the applied bias spatially separates the charges and thus reduces the electron-hole exchange coupling. As a result, the interaction of the hole spin with phonons becomes stronger than the exchange interaction, and this leads to a breakage of the coupling between the electron and hole spins. As a consequence, the hole spin exhibits fast relaxation, while the electron spin holds its orientation, providing the slow component in the PL polarization decay. The decay time $\tau=1 \mathrm{~ns}$ measured at $U_{\text {bias }}$ $=-2 \mathrm{~V}$ well agrees with the literature data on the relaxation time of the electronic spin in the GaAs-based heterostructures. $^{24}$

The possibility to break the spin coupling strongly depends on the QW thickness. With decreasing thickness of the QW, the exchange interaction increases and the hole spin relaxation rate decreases. In addition, in thin QWs, the efficiency of spatial separation of the electron and hole by the electric field is smaller than in thick wells. ${ }^{17}$ Due to the joint action of these factors, a relatively small change in the QW thickness (from 10 to $8 \mathrm{~nm}$ ) drastically increases the electric field required for breakage of the coupling of the electron and hole spins. This fact explains the absence of noticeable changes in the polarized PL kinetics of the samples with the $8 \mathrm{~nm}$ QWs in the whole range of the bias used in our experiments.

The threshold-like nature of the competition between the exchange and spin-phonon interaction is revealed in the kinetics of the degree of PL circular polarization of the $10 \mathrm{~nm}$ QWs under intermediate values of the electric bias. As was noted in Sec. III A, the change in the bias leads to a redistribution of amplitudes of the slow and fast components, with their time constants remaining the same. We suppose that the reason for this redistribution is related to the inhomogeneity of the excitonic ensemble in the QWs which may be caused both by the local variations of the QW thickness and by random electric fields created by charged impurities. Due to the inhomogeneity, the critical values of the electric fields breaking the electron-hole spin coupling have a certain spread and, at small applied biases, are realized only for a fraction of the localized excitonic states. As the bias increases, this fraction grows, which is revealed as an increase of the slow component.

\section{Polarization dynamics in the transverse magnetic field}

An analysis of the PL polarization dynamics in a transverse magnetic field gives additional evidence for the exchange interaction suppression in an external electric field. In the presence of the bias, the hole spin rapidly relaxes and its projection onto the direction of observation varies in a random way between $+3 / 2$ and $-3 / 2$. Being uncoupled with the hole spin, the electronic spin may freely precess around the magnetic field direction. The projection of the electron 
spin onto the direction of observation will oscillate in time between $-1 / 2$ to $+1 / 2$ with the frequency $\omega=g_{e} \mu B / \hbar$, where $g_{e}$ is the electron $g$ factor, $\mu$ is the Bohr magneton, $B$ is the magnetic field strength, and $\hbar$ is the Planck constant. The degree of circular polarization should oscillate between +1 and -1 with the same frequency because, when the hole spin has no preferential orientation, this quantity is determined only by the projection of the electron spin. ${ }^{25}$ The oscillations of the degree of polarization, observed under the bias $U_{\text {bias }}=-2 \mathrm{~V}$ [lower curve in Fig. 2], completely correlate with this scenario. The oscillations are symmetric with respect to the $x$ axis and their frequency is proportional to the magnetic field strength [Fig. 3(a)] with the proportionality factor corresponding to the value $g=0.21 \pm 0.02$. This well agrees with the experimental and theoretical estimates of the transverse electron $g$-factor in the GaAs QWs of the same thickness. ${ }^{26,27}$

In the absence of the bias, one has to consider the behavior of the exciton spin as a whole. The transverse magnetic field mixes the states of the excitonic quartet and, as a result, the $\sigma^{+}$polarized light becomes capable of exciting several states. The kinetics of the polarized PL is controlled, in this case, by the interference of the states of the exciton fine structure split by the combined action of the magnetic field and exchange coupling. ${ }^{28,29}$ The degree of polarization should vary in time in a rather complicated fashion, reflecting superposition of the beats at several frequencies. ${ }^{30}$ The upper curve in Fig. 2 demonstrates exactly this kind of the polarization kinetics.

A detailed analysis of the exciton spin dynamics lies outside the scope of this paper. We can only note here that the lowest of the beat frequencies should correlate, in this case, with the splitting of the heavy-hole spin states, characterized by extremely low value of the transverse $g$-factor. ${ }^{31}$ Indeed, the oscillation frequencies obtained from the experimental data increased linearly with the magnetic field strength [Fig. 3(a), lower curve], with the proportionality factor corresponding to the $g=0.06 \pm 0.01$. This value virtually coincides with the transverse component of the heavy-hole $g$-factor $\left(g_{h x}=0.04 \pm 0.01\right)$, found experimentally in Ref. 30 for the structures with close parameters. Thus, the dynamics of the polarized PL in the transverse magnetic field in the absence of the bias well agrees with the suggestion that this dynamics is controlled by the exciton spin.

For intermediate values of the bias, the breakage of the exchange coupling is realized only for a fraction of the states of the inhomogeneous excitonic ensemble (see Sec. III B 1). In this case, the kinetics of the degree of circular polarization should represent a combination of the slowly and rapidly damping oscillating components, which is really observed experimentally [Fig. 2].

The experimental data presented in this section allow us to conclude that application of the bias to the structures with $10 \mathrm{~nm}$ quantum wells weakens the exchange interaction between the electron and hole spins. As a result, the hole spin rapidly relaxes while the electron spin holds the lightinduced orientation for a fairly long time and, in the presence of a magnetic field, may freely precess around its axis. Therefore, the use of the external electric bias makes it pos-
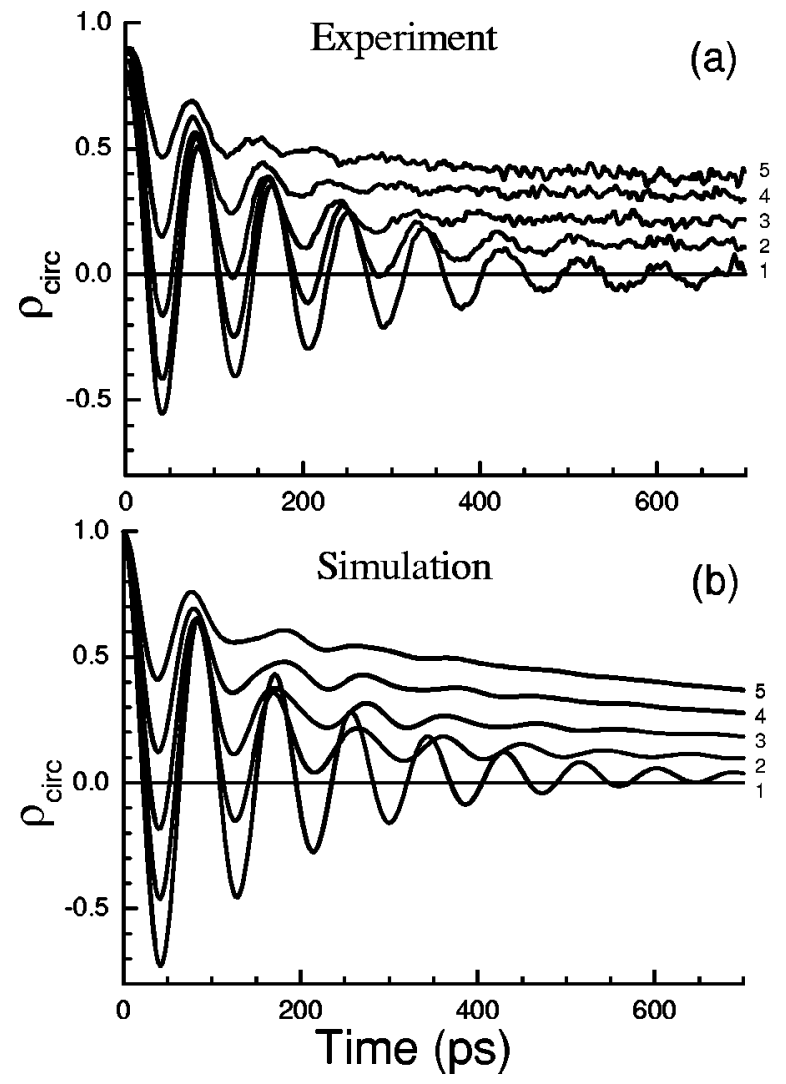

FIG. 4. The kinetics of the degree of circular polarization of the PL of the $10 \mathrm{~nm}$ MQWs in oblique magnetic fields. $(B=4 \mathrm{~T})$ : (a) experimental curves, (b) results of calculations. The values of the tilt angles: $1-\theta=90^{\circ} ; 2-\theta=110^{\circ} ; 3-\theta=120^{\circ} ; 4-\theta$ $=130^{\circ} ; 5-\theta=140^{\circ}$.

sible to study the free-electron spin dynamics even in the structures where the electron and hole spins are initially strongly coupled. In Sec. IV we will show that this approach enables one to get new information about the spin relaxation of electrons.

\section{ELECTRON-SPIN DYNAMICS IN OBLIQUE MAGNETIC FIELDS}

In this section we analyze the data about kinetics of the polarized PL in the $10 \mathrm{~nm}$ QWs at the bias $U_{\text {bias }}=-2 \mathrm{~V}$, i.e., under conditions when the PL polarization is determined by the electron spin orientation. We studied the dependence of the electron spin relaxation rate on the magnitude and orientation of the external magnetic field.

\section{A. Experimental data}

The dependence of the oscillation decay rate $\tau^{-1}$ on the magnetic field strength in the Voigt configuration is shown in Fig. 3(b). The values of the decay rates were obtained by fitting the experimentally measured oscillations with Eq. (1). As seen from the figure, the increase of the magnetic field is accompanied by practically linear growth of the beat decay rate. Extrapolation of the linear dependence to zero field 

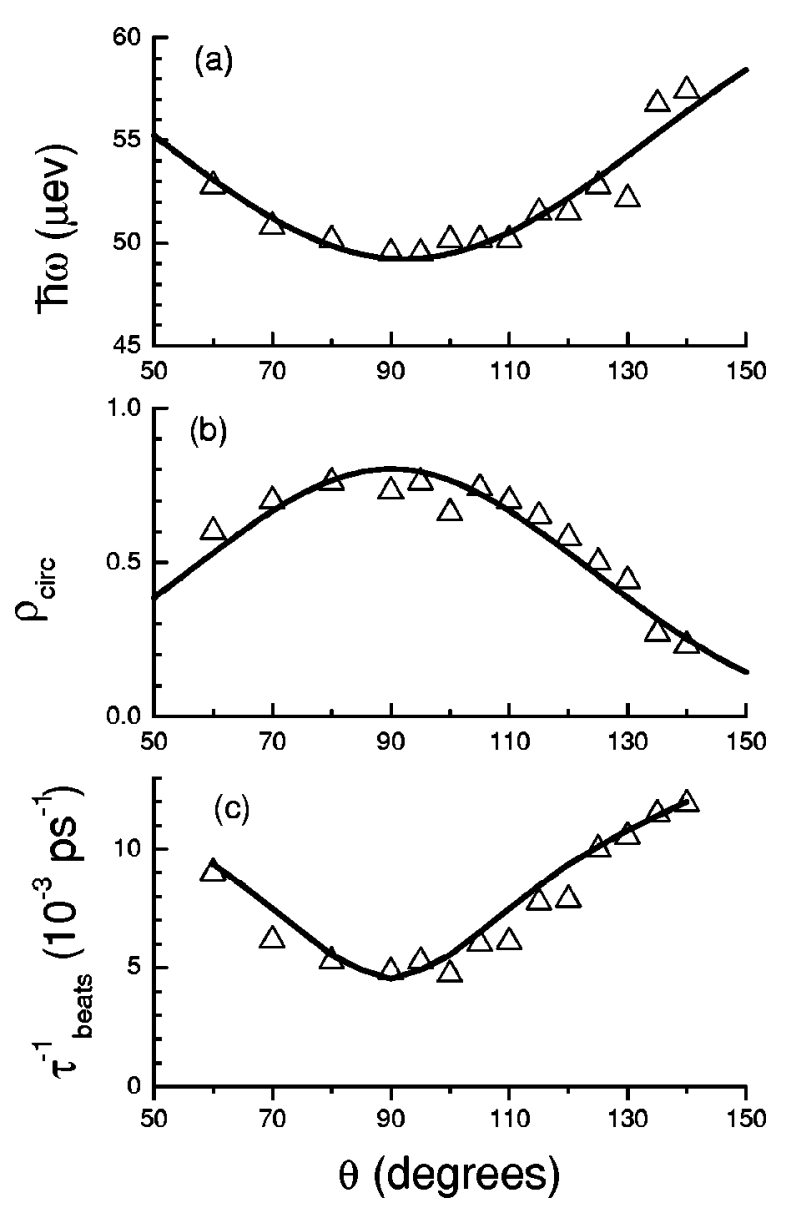

FIG. 5. Characteristics of the oscillations of the degree of circular polarization of the PL of the $10 \mathrm{~nm}$ MQWs in oblique magnetic fields. (a) Variation of the oscillation frequency $\omega$ with the tilt angle $\theta$. Open triangles are experimental values, solid line is the fitting by formula (2). The values of the fitting parameters $g_{z}$ $=0.26$ and $g_{x}=0.21$. (b) Variation of the oscillation amplitude $\rho_{0}^{\text {osc }}$ with the tilt angle $\theta$. Open triangles are experimental values, solid line is the fitting by formula (4). (c) Variation of the oscillation decay rate $\tau^{-1}$ with the tilt angle $\theta$. Open triangles are experimental values, solid line is the result of calculations (see the text).

yields $\tau^{-1}=(8.5 \pm 1) \times 10^{-4} \mathrm{ps}^{-1}$, which is close to the decay rate of the degree of circular polarization measured with no magnetic field [see Fig. 1].

Figure 4(a) shows variations in the dynamics of circular polarization with the magnetic field direction. When the angle $\theta$ deviates from $\theta=90^{\circ}$, a non-oscillating weakly damping component (pedestal) arises, with its amplitude growing with the deviation angle. Simultaneously, the amplitude of the oscillating component, $\rho_{0}^{\text {osc }}$, decreases. The analysis of the oscillating part of the signal has shown that it can be well approximated by the damping oscillating function of the type (1). This allowed us to find the dependencies of the amplitude $\rho_{0}^{\text {osc }}$, frequency $\omega$, and decay rate of the oscillations $\tau^{-1}$ on the angle $\theta$. In these dependencies shown in Fig. 5 one can distinctly see that the deviation from the exact Voigt configuration $\left(\theta=90^{\circ}\right)$ is accompanied by a slight increase in the oscillation frequency with a substantial increase in their decay rate.

\section{B. Discussion \\ 1. Angular dependencies of the oscillation amplitude and frequency}

The changes in the frequency and amplitude of the oscillations in oblique magnetic fields are quite understandable. The increase in the oscillation frequency upon deviation of the magnetic field from the transverse direction [see Fig. $5(\mathrm{a})]$ is related to the anisotropy of the electron $g$-factor resulting from the size quantization effect. According to the theory (Ref. 23), the splitting between the spin sublevels of the free electron in quasi-2D structures is given by

$$
\Delta E=\mu B \sqrt{\left(g_{z} \cos \theta\right)^{2}+\left(g_{x} \sin \theta\right)^{2}},
$$

where $g_{z}$ and $g_{x}$ are the longitudinal and transverse components of the electron $g$-factor. By forcing the experimental data to fit Eq. (2) [solid line in Fig. 5(a)] we have found the values of the corresponding components in the structure under study: $g_{z}=0.26$ and $g_{x}=0.21$. These values well agree with the values $g_{z}=0.24$ and $g_{x}=0.19$ given in Ref. 27 for the QWs of the same thickness, as well as with the results of the theoretical calculations. ${ }^{26,32}$

The experimental dependence of the initial oscillation amplitude $\rho_{0}^{\text {osc }}$ on the angle $\theta$ between the magnetic field and the direction of observation [Fig. 5(b)] can also be easily explained. The PL polarization is determined by the orientation of the electron spin with respect to the direction of observation. The optical excitation aligns the electron spin along the $z$ axis. When the electron spin precesses around the transverse magnetic field $\left(\theta=90^{\circ}\right)$, its projection onto the direction of observation periodically changes the sign, which leads to the oscillations of the degree of polarization. In the longitudinal magnetic field $\left(\theta=0^{\circ}\right)$, the projection of the electron spin onto the direction of observation does not change with time and, therefore, the degree of polarization remains constant. For intermediate values of the angle $\theta$, the degree of circular polarization contains both the oscillating and non-oscillating components. The theoretical calculation of the degree of polarization of the PL $\rho$, performed with the use of the model electron spin-Hamiltonian built on the basis functions $|+1 / 2\rangle$ and $|-1 / 2\rangle,{ }^{16}$ yields, in the general case (with no allowance for the damping), the following expression for $\rho$ :

$$
\rho=\rho_{0}^{\mathrm{osc}} \cos (\omega t)+\rho_{0}^{\mathrm{sm}} .
$$

The amplitudes of the oscillating $\rho_{0}^{\text {osc }}$ and smooth $\rho_{0}^{\text {sm }}$ components in Eq. (3) are controlled by the transverse $\left(B_{x}\right.$ $=B \sin \theta)$ and longitudinal $\left(B_{z}=B \cos \theta\right)$ components of the magnetic field:

$$
\begin{gathered}
\rho_{0}^{\mathrm{osc}}=\frac{\rho_{0}\left(\mu g_{x} B_{x}\right)^{2}}{\left(\mu g_{x} B_{x}\right)^{2}+\left(\mu g_{z} B_{z}\right)^{2}}=\frac{\rho_{0}\left(g_{x} \sin \theta\right)^{2}}{\left(g_{x} \sin \theta\right)^{2}+\left(g_{z} \cos \theta\right)^{2}}, \\
\rho_{0}^{\mathrm{sm}}=\frac{\rho_{0}\left(\mu g_{z} B_{z}\right)^{2}}{\left(\mu g_{x} B_{x}\right)^{2}+\left(\mu g_{z} B_{z}\right)^{2}}=\frac{\rho_{0}\left(g_{z} \cos \theta\right)^{2}}{\left(g_{x} \sin \theta\right)^{2}+\left(g_{z} \cos \theta\right)^{2}} .
\end{gathered}
$$


As is seen from Fig. 5(b), the theoretical curve given by Eq. (4) agrees well with the experimental data with the fitting parameter $\rho_{0}=0.8$.

\section{The decay of the oscillations in the Voigt configuration}

The data on variations of the oscillation decay time with experimental conditions should be discussed separately because they contain essential information about relaxation processes in the spin system. Since the circular polarization of the PL is determined by the electron spin orientation, the only process that destroys the PL polarization in the absence of the magnetic field is the energy relaxation of the electron spin (spin flips). It is exactly this process that controls the polarization decay time measured in zero magnetic field at $U_{\text {bias }}=-2 \mathrm{~V}(\tau \approx 1 \mathrm{~ns}$, see Sec. III A). The energy relaxation of the electron spin should also lead to a decay of the polarization oscillations in the transverse magnetic field. Indeed, approximately the same time $\tau \approx 1$ ns characterizes the oscillation decay detected in small magnetic fields.

As the magnetic field increases, the decay rate of the oscillations also increases, i.e., the transverse magnetic field induces an additional spin relaxation process. We suppose that the additional decay is caused by the inhomogeneous broadening of the oscillation spectrum $\left(\Delta \omega_{B}\right)$ originating from a spread of the electron $g$-factor in the excitonic ensemble. Because of the spread of the frequencies, the coherence of the oscillations created by the exciting pulse in the excitonic ensemble is gradually destroyed, and the total amplitude of the oscillating signal decreases with time (reversible dephasing). ${ }^{9,33}$ If the spread of the electron $g$-factor obeys the Gaussian distribution function with the halfwidth $\Delta g_{e}$, the oscillation decay time is given by the relationship $\gamma_{B} \approx \Delta \omega / 2=\Delta g_{e} \mu B / 2$. Indeed, as is seen from Fig. 3(b), the decay rate linearly varies with the field, which is a convincing evidence in favor of the proposed mechanism of the decay. The spread of the electron $g$-factor evaluated from the slope of the line in Fig. 3(b) comprises $\Delta g_{e} \approx 0.02$, which makes approximately $10 \%$ of the $g$-factor absolute value.

\section{Angular dependence of the oscillation decay rate}

A fundamentally new result is a substantial growth of the oscillation decay rate upon deviation of the magnetic field from the exactly transverse direction. As seen from Fig. 5(c), the change of the angle from $90^{\circ}$ to $140^{\circ}$ is accompanied by more than twofold increase in the decay rate. It should be emphasized that, according to the conclusions of the previous subsection, in the magnetic field $B=4 \mathrm{~T}$ used for studying the angular dependence, the decay of the oscillations is mainly related to the spread of the electron $g$-factor rather than to the electron spin flips. Thus, the observed effect cannot be explained by the electron-spin relaxation rate anisotropy discussed in Refs. 34 and 35.

The reason for the change in the decay rate under variation of the field direction may be related, to a certain extent, to a difference in the spread of the longitudinal and transverse components of the electron $g$-factor. However, to explain the experimental data quantitatively, one has to suppose that the spread of the longitudinal component is nearly by a factor of 4 larger than that of the transverse component. Since these components are close in magnitude (see Sec. IV B 1), the possibility of such a difference in the spread of their values looks rather unlikely.

From our point of view, the reason for the additional decay of the oscillations is related to the residual exchange interaction between the electron spin and rapidly relaxing spin of the hole. The exchange interaction can be regarded as an effective magnetic field acting on the electron spin. The field is directed along the spin of the hole, i.e., is parallel to the $z$ axis. The speed and direction of the electron spin precession are determined by the total field $B^{\text {sum }}$, representing a vector sum of the magnetic and exchange fields. The magnitude and direction of the exchange field are determined by the state of the hole spin, which is in the general case, a linear combination of the states with opposite spin directions, i.e., $m_{h}=a|+3 / 2\rangle+b|-3 / 2\rangle$. The coefficients $a$ and $b$ obey the normalization condition $\sqrt{a^{2}+b^{2}}=1$. The $\sigma_{+}$polarized excitation creates the hole spin state with $a=1$ and $b=0$. After the hole spin is relaxed, the coefficients $a$ and $b$ acquire random values, and the mean value of the exchange field vanishes, with its instantaneous values varying in time in a random way. As a result, a fluctuating component arises in the total field acting on the electron spin. This component broadens the frequency spectrum of the oscillations and thus accelerates the oscillations decay. In other words, the exchange interaction with the disoriented hole spin is accompanied by the irreversible phase relaxation of the electron spin. This results in an additional damping of the oscillations of the degree of polarization.

The contribution of the exchange interaction into this damping depends on mutual orientation of the exchange and magnetic fields. Since the direction of the exchange field is parallel to the growth axis of the structure, the oscillation frequency under arbitrary orientation of the magnetic field can be determined by

$$
\omega_{h}=\frac{1}{\hbar} \mu \sqrt{g_{x}^{2}(B \sin \theta)^{2}+g_{z}^{2}\left(B_{h}+B \cos \theta\right)^{2}},
$$

where $B_{h}=\delta_{h} /\left(g_{e} \mu\right)$ is the instantaneous value of the fluctuating exchange field corresponding to the exchange splitting $\delta_{h}$. As is seen from Eq. (6), the effect of the exchange field on the oscillation frequency may substantially differ for different orientations of the external magnetic field.

In the exact Voigt configuration, with $\theta=90^{\circ}$, the oscillation frequency is determined by the sum of squares of the external and exchange fields:

$$
\omega_{h}=\frac{1}{\hbar} \mu \sqrt{g_{x}^{2} B^{2}+g_{z}^{2} B_{h}^{2}} .
$$

In this case, the decay of the oscillations, related to the exchange field fluctuations, may be effective only in low magnetic fields when $B<B_{h}$.

When deviating from the Voigt configuration, a longitudinal component of the magnetic field arises, which is linearly 
summed up with the exchange field [see Eq. (6)]. As a result, the contribution of the fluctuating component to the total field increases, and this should lead to an increase in the frequency fluctuations and, correspondingly, to an increase in the oscillation decay rate.

To estimate the efficiency of such a process in quantitative terms, we have made model calculations of the kinetics of the degree of PL polarization for different values of the tilt angle $\theta$. The procedure of the calculations and their results are presented in the next section.

\section{Model calculations of the kinetics of degree of polarization}

The degree of polarization of the PL excited by a $\delta$-wise light pulse was calculated as a sum of the oscillating and smooth terms in accordance with Eq. (3). The amplitudes of these terms and the oscillation frequencies were calculated for each value of the exchange field $B_{h}$. Then, the total shape of the signal was averaged over the spectrum of the random exchange-field values $F\left(B_{h}\right)$, realized in the process of the hole spin relaxation.

The final formula used for the calculations had the form:

$$
\begin{aligned}
\rho(t, \theta)= & \int F\left(B_{h}\right)\left[\rho_{0}{ }^{\mathrm{osc}}\left(\theta, B_{h}\right) e^{-\left(\gamma_{0}+\gamma_{B}\right) t} \cos \left(\omega_{h} t\right)\right. \\
& \left.+\rho_{0}{ }^{\mathrm{sm}}\left(\theta, B_{h}\right) e^{-\gamma_{0} t}\right] d B_{h} .
\end{aligned}
$$

The amplitudes of the oscillating and smooth components $\rho_{0}^{\text {osc }}\left(\theta, B_{h}\right)$ and $\rho_{0}^{\text {sm }}\left(\theta, B_{h}\right)$ are given by equations which are obtained from Eqs. (4) and (5) by replacing of the external magnetic field with the acting field $B_{x}^{\text {sum }}=B \sin \theta$ and $B_{z}^{\text {sum }}$ $=B \cos \theta+B_{h}$. The frequency $\omega_{h}$ is defined by Eq. (6). The exponential factor in the first term of formula (8) describes the decay of the oscillations related to the electron spin flips (constant $\gamma_{0}$ ) and to a spread of the electron $g$-factor (constant $\gamma_{B}$ ). The exponent of the second term in Eq. (8) does not contain $\gamma_{B}$, because the spread of the electron $g$-factor should not affect the decay of PL polarization in the longitudinal field.

The values of $\gamma_{0}=8.5 \times 10^{-1} \mathrm{ps}^{-1}$ and $\gamma_{B}=\Delta g_{e} \mu B / 2$ $=3.6 \times 10^{-3} \mathrm{ps}^{-1}$ (for $B=4 \mathrm{~T}$ ) were found from the dependence of the oscillation decay rate on the field in the Voigt configuration [see Fig. 3(b)]. The value $\rho_{0}=0.8$ was determined in Sec. IV B 1 using the angular dependence of the oscillation amplitude. The values $g_{x}=0.21$ and $g_{z}=0.26$ were obtained in Sec. IV B 1 from the analysis of the angular dependence of the oscillation frequencies. In that analysis, we did not take into account the effect of the exchange field on the mean oscillation frequency. For more precise calculations of $\rho(t, \theta)$, we used the components of the electronic $g$-factor as fitting parameters. It was found, however, that the values thus obtained virtually coincided with those given above. This result is quite understandable from a physical point of view. The oscillation frequency is determined by the time-averaged value of the effective magnetic field. The mean value of the fluctuating exchange field is zero, so this field does not noticeably affect the averaged effective field.

Thus, in our calculations, the main variable parameters were the range of integration and the functional form of the factor $F\left(B_{h}\right)$ which determined the spectrum of the effective exchange field. Having no prior idea about the shape of this spectrum, we assumed that the values of the exchange field are uniformly distributed within the range $\left(-B_{h}^{0},+B_{h}^{0}\right)$ and used the quantity $B_{h}^{0}$ as a fitting parameter. The test calculations with other functions $F\left(B_{h}\right)$ (e.g., triangular or Gaussian) have shown that, except for small variations in the value of $B_{h}^{0}$, no essential changes in the final results are noticed.

Figure 4(b) shows the kinetics of the degree of circular polarization calculated for several angles $\theta$ using a fixed value of the exchange field $B_{h}^{0}=1 \mathrm{~T}$. One can see that the calculated curves perfectly reproduce all the changes arising in the kinetics of the degree of circular polarization upon deviation of the external magnetic field from the exact transverse direction.

The oscillating parts of the theoretical curves were fitted by Eq. (1). The decay rates $\tau^{-1}$, thus determined, were compared with those obtained experimentally. The results of this comparison, presented in Fig. 5(c), demonstrate good agreement between the theory and experiment.

The calculated value of the effective exchange field $B_{h}^{0}$ $=1 \mathrm{~T}$ corresponds to the exchange splitting $\delta_{h}=B_{h}^{0} / g_{z} \mu$ $=16 \mu \mathrm{eV}$. This value is substantially smaller than typical values of the exchange splitting in excitonic states in the GaAs QWs: $\delta_{0}=100 \div 150 \mu \mathrm{eV} .{ }^{9,10,36}$ It is natural to suggest that the main reason for this difference is the suppression of the exchange interaction by the electric field. The value of difference demonstrates the efficiency of the suppression in the $10 \mathrm{~nm} \mathrm{GaAs}$ QWs.

\section{CONCLUSION}

A theoretical possibility to affect the spin dynamics of the carriers in quantum-confined semiconductor structures by means of electric field has been already discussed in the literature. ${ }^{16,17}$ According to Ref. 16, upon resonance excitation of the excitonic states, the processes of independent relaxation of the electron and hole spins are not efficient, and the electric field may change only the relaxation rate of the exciton spin as a whole. In this case, an increase in the field strength should result in a smooth decrease of the relaxation rate. The results of our studies show that this is not the case. The electric field may switch, in a threshold way, the dynamics of the exciton spin to that of independent electron and hole spins, with essentially different parameters. Thus, the use of the external electric bias allows one to efficiently control the spin dynamics of carriers in the structures with the GaAs QWs. It is not improbable that, in the future, the discovered effect will find its application in the systems of spintronics and spin information processing.

In the studies of kinetics of the degree of polarization of PL in an oblique magnetic field, we have found a new process of electron spin dephasing related to the exchange interaction with the relaxing spin of the hole. The model calculation of the polarization kinetics confirmed the correctness of the interpretation of the experimental data and allowed us to estimate the degree of suppression of the electron-hole ex- 
change interaction by the external electric field. This estimate can be considered as unique because, under conditions of large homogeneous broadening caused by fast relaxation of the hole spin, the exchange splitting cannot be measured by direct spectroscopic methods as, e.g., ODMR or levelantiscrossing spectroscopy.

\section{ACKNOWLEDGMENTS}

The authors are grateful to Professor V. Zapasskii for the critical reading of the manuscript. This work is supported by CRDF, Grant No. RP2-2356-ST-02 and by RFBR, Project No. 02-02-16459.

${ }^{21}$ J.G. Tischler, A.S. Bracker, D. Gammon, and D. Park, Phys. Rev. B 66, 081310 (2002).

${ }^{22}$ H.-J. Polland, L. Schultheis, J. Kuhl, E.O. Gobel, and C.W. Tu, Phys. Rev. Lett. 55, 2610 (1985).

${ }^{23}$ H.W. van Kesteren, E.C. Cosman, W.A.J.A. van der Poel, and C.T. Foxon, Phys. Rev. B 41, 5283 (1990).

${ }^{24}$ R.I. Dzhioev, V.L. Korenev, B.P. Zakharchenya, D. Gammon, A.S. Bracker, J.G. Tischler, and D.S. Katzer, Phys. Rev. B 66, 153409 (2002).

${ }^{25}$ V.K. Kalevich, B.P. Zakharchchenya, K.V. Kavokin, A.V. Petrov, P. Le Jeune, X. Marie, D. Robart, T. Amand, J. Barrau, and M. Brouseau, Fiz. Tverd. Tel. 39, 768 (1997) [Phys. Solid State 39, 681 (1997)].

${ }^{26}$ E.L. Ivchenko and A.A. Kiselev, Fiz. Tekh. Poluprovodn. (St.Petersburg) 26, 1471 (1992) [Sov. Phys. Semicond. 26, 827 (1992)].

${ }^{27}$ A. Malinowski and R.T. Harley, Phys. Rev. B 62, 2051 (2000).

${ }^{28}$ M. Dyakonov, X. Marie, T. Amand, P. Le Jeune, D. Robart, M. Brousseau, and J. Barrau, Phys. Rev. B 56, 10412 (1997).

${ }^{29}$ D. Hagele, J. Hubner, W.W. Ruhle, and M. Oestreich, Solid State Commun. 120, 73 (2001).

${ }^{30}$ I.A. Yugova, I.Ya. Gerlovin, V.G. Davydov, I.V. Ignatiev, I.E. Kozin, H.W. Ren, M. Sugisaki, S. Sugou, and Y. Masumoto, Phys. Rev. B 66, 235312 (2002).

${ }^{31}$ X. Marie, T. Amand, P. Le Jeune, M. Paillard, P. Renucci, L.E. Golub, V.D. Dymnikov, and E.L. Ivchenko, Phys. Rev. B 60, 5811 (1999).

${ }^{32}$ E.L. Ivchenko, A.A. Kiselev, and M. Willander, Solid State Commun. 102, 375 (1997).

${ }^{33}$ H. Nickolaus, H.-J. Wunsche, and F. Henneberger, Phys. Rev. Lett. 81, 2586 (1998).

${ }^{34}$ Y. Ohno, R. Terauchi, T. Adachi, F. Matsukura, and O.H. Ohno, Phys. Rev. Lett. 83, 4196 (1999).

${ }^{35}$ N.S. Averkiev, L.E. Golub, and M. Willander, J. Phys.: Condens. Matter 14, R271 (2002).

${ }^{36}$ E. Blackwood, M.J. Snelling, R.T. Harley, S.R. Andrews, and C.T.B. Foxon, Phys. Rev. B 50, 14246 (1994). 